\title{
Examining Outcome Variability: Correlates of Treatment Response in a Child and Adolescent Anxiety Clinic
}

\author{
Michael A. Southam-Gerow \\ Department of Psychology, University of California-Los Angeles \\ Philip C. Kendall \\ Department of Psychology, Temple University \\ V. Robin Weersing \\ Department of Psychology, University of California-Los Angeles
}

\begin{abstract}
Examined correlates of treatment response in a clinic providing cognitive-behavioral therapy for children with anxiety disorders. Youth (ages 7 to 15) with a primary Diagnostic and Statistical Manual of Mental Disorders (3rd ed., rev., or 4th ed.; American Psychiatric Association, 1987, 1994) anxiety-disorder diagnosis (overanxious disorder, generalized anxiety disorder, separation anxiety disorder, social phobia, or avoidant disorder) participated. After completing a full course of treatment and posttreatment $(n=135)$ and 1 -year follow-up $(n=107)$ assessments, participants were classified into 1 of 2 groups - poor treatment response and good treatment responseusing parent diagnostic reports. Discriminant function analyses indicated that higher levels of maternal-and teacher-reported child-internalizing psychopathology at pretreatment, higher levels of maternal self-reported depressive symptoms, and older-child age were all associated with less favorable treatment response. Other factors, such as child ethnicity, child sex, family income, family composition (i.e., dual parent vs. single parent), child-reported symptomatology, and maternal-reported level of childexternalizing behavior problems did not predict treatment response. Both practical and conceptual implications of the findings are discussed.
\end{abstract}

Results from controlled clinical trials and metaanalyses suggest that for many children with mental health problems, psychotherapy can be beneficial (Casey \& Berman, 1985; Kazdin, Bass, Ayers, \& Rodgers, 1990; Weisz, Weiss, Han, \& Granger, 1995). However, these generally positive results are tempered by less favorable outcomes for subsamples of children who seek mental health services. For example, recent research on treatment "dropouts" and work examining poor outcome despite a full course of treatment has

Michael A. Southam-Gerow is now at Virginia Commonwealth University. V. Robin Weersing is now at Western Psychiatric Institute and Clinic, Pittsburgh, PA.

This study was supported in part by National Institute of Mental Health Grants R01-MH44042 and R01-MH57347.

An earlier version of this article was presented as a poster at the Kansas Conference on Clinical Child Psychology, Lawrence, KS, October 1998

We thank our colleagues at the Child and Adolescent Anxiety Disorders Clinic at Temple University. We also express our appreciation to the families who agreed to participate in the study.

Requests for reprints should be sent to Michael A. SouthamGerow, Department of Psychology, Virginia Commonwealth University, 808 W. Franklin Street, Box 842018, Richmond, VA 23284-2018. demonstrated that for some youth, treatment response is minimal. Indeed, even in randomized clinical trials that support the use of a particular treatment, a portion of the children who have completed treatment continue to experience marked psychosocial impairment. For example, Weisz et al. found that, on average, approximately $22 \%$ of treated youth fared worse than the average member of the no-treatment control groups. There are both practical and conceptual reasons to study poor treatment response. Practically speaking, as our knowledge of the factors associated with treatment response for particular treatments increases, so does our ability to assign children to appropriate treatment approaches (i.e., in an empirically prescriptive manner). For example, one treatment may prove most helpful in cases with high levels of comorbidity; whereas, for children with fewer disorders, a less intensive approach may prove sufficient. In addition, knowledge of the relevant response-related factors will inform decisions about supplementing or modifying current treatment approaches. For example, if parental psychopathology is associated with poor treatment response (e.g., Patterson \& Chamberlain, 1994), concurrent family therapy or adjunctive individual therapy for the parent may be indicated. 
From a more conceptual viewpoint, the study of poor treatment response has the potential to strengthen the field's understanding of psychopathology and psychotherapy. Knowledge of variables that predict response to a particular treatment may help define the efficacy "boundaries" of particular treatment approaches. For example, some have argued that a certain level of cognitive development is considered integral for cognitive therapy (CT) to be most beneficial (e.g., Durlak, Fuhrman, \& Lampman, 1991; Weisz \& Weersing, 1998). If CT is less effective for younger children than another therapy (e.g., behavior therapy), then the proposed developmental boundary of CT receives empirical support.

To date, the majority of research on treatment response has been conducted in the area of conduct disorders in youth (typically parent-training programs; e.g., Dumas, 1984; Patterson \& Chamberlain, 1994; Webster-Stratton, 1985). Several factors have been found to be associated with the poorest outcomes: (a) olderchild age (e.g., Patterson \& Chamberlain, 1994); (b) higher levels of parental psychopathology (e.g., Patterson \& Chamberlain, 1994); (c) higher levels of marital-parental-relational discord (e.g., Dadds, Schwartz, \& Sanders, 1987); (d) lower levels of family socioeconomic status (e.g., Patterson \& Chamberlain, 1994); (e) higher levels of parental social isolation and limited social support (i.e., the insular parent; see Dumas \& Wahler, 1983); (f) single-parent households (e.g., Webster-Stratton, 1985); (g) lower levels of therapist training and involvement (e.g., Bush, Glenwick, \& Stephens, 1986); (h) poorer treatment adherence or fidelity (e.g., Henggeler, Melton, Brondino, Scherer, \& Hanley, 1997); and (i) lower levels of family investment in treatment (e.g., Nye, Zucker, \& Fitzgerald, 1995). Patterson and colleagues also found that failure in their parent-training program was predicted by chronic or intense parental resistance to treatment (see Patterson \& Chamberlain, 1994; Stoolmiller, Duncan, Bank, \& Patterson, 1993).

Empirical work on factors related to treatment response for children with internalizing disorders has been scanty. There were only three studies we found that specifically examined predictors of poor treatment response for children with internalizing problems, and all three focused on depressed adolescents. Clarke et al. (1992) examined several treatment-response predictors (e.g., age, sex, components of therapy, family composition) in a study of adolescents treated with a cognitive-behavioral group approach. Using a categorical definition of treatment response (i.e., recovered vs. not recovered, based on adolescent-reported diagnostic information), they found that higher adolescent self-reported Beck Depression Inventory (BDI) scores, higher adolescent self-reported state anxiety scores, lower adolescent self-reported enjoyment of and frequency of pleasant activities, and fewer adolescent self-reported rational thoughts were associated with poorer outcomes.

Two recent studies reported findings largely consistent with Clarke et al.'s (1992) findings. First, in a study of the treatment of depressed children and adolescents (ages 10 to 17) using cognitive-behavioral therapy (CBT), Jayson, Wood, Kroll, Fraser, and Harrington (1998) found that older child age and higher levels of child self-reported depression severity predicted the poorest outcomes as defined categorically by remission of major depressive disorder (reported by the youth on the Schedule for Affective Disorders and Schizophrenia-School Age Version [K-SADS]). In a sample of depressed teens treated with psychosocial treatments (e.g., CBT, systemic-behavioral family therapy), Brent et al. (1998) examined predictors of three separate categorical treatment-response indicators: (a) continued depressive episode (using K-SADS combined parent-child report), (b) remission (using a cutoff on adolescent-reported BDI scores), and (c) poor functional status (using a cutoff score of clinician-rated Children's Global Assessment Scale scores). They found that symptom severity across reporters (i.e., child, parent, interviewer) predicted poorest outcomes, with clinical referral (vs. response to advertisements) and higher levels of cognitive distortion and hopelessness predicting a continued episode, higher levels of self-reported depression predicting remission, and higher levels of interviewer-rated depression predicting poor functional status. Overall, the evidence, though sparse, indicates that symptom severity and cognitive variables (e.g., cognitive distortion, fewer rational thoughts) are the most consistent predictors of poor treatment response for depressed teens.

This study focused on predictors of treatment response ${ }^{1}$ for children who received a full course of treatment (i.e., at least 12 sessions) at a child and adolescent anxiety disorders clinic. We operationalized treatment response categorically, with poor response defined by the presence of one or more anxiety disorders at the posttreatment and 1-year follow-up assessments. Because research has suggested that parent-child diagnostic agreement is low (e.g., Frick, Silverthorn, \& Evans, 1994; Stanger \& Lewis, 1993), we considered dividing the sample twice-once using parent-reported diagnoses and once using child-reported diagnoses.

\footnotetext{
${ }^{1}$ We did not consider factors associated with attrition from therapy (for review, see Armbruster \& Kazdin, 1994) and thus did not include youth who left treatment prematurely. We made this choice because dropout may be predicted by a different set of variables than those related to treatment response after a complete protocol. For example, an earlier study in our lab (Kendall \& Sugarman, 1997) found that ethnicity and family composition variables were related to attrition, with non-Caucasian youth and single-parent families more likely to drop out of treatment. Symptom severity was also related to attrition, but those with the most severe symptoms remained in treatment.
} 
However, we rejected this course of action and relied solely on parent-report diagnostic categories for two reasons. First, parents referred all participating children to our clinic. As such, parent perception of child problems was, by definition, the primary reason for seeking therapeutic services, thereby representing the best index for treatment response. A second and related point was that a parent-reported child anxiety-disorder diagnosis was the primary inclusion criterion for participants in this study and in the clinical trials (see Kendall, 1994; Kendall et al., 1997). Thus, focusing only on parent-reported diagnoses was deemed most appropriate for project aims and characteristics of this sample. ${ }^{2}$

We examined the association of several factors with treatment response. We included several child symptom variables: (a) severity of child psychopathology (e.g., anxiety, depression, conduct problems) as judged by child, mother, and teacher; (b) cognitive symptoms judged by child; and (c) number of comorbid diagnoses, along with three child characteristic variablesage, gender, and ethnicity. In addition, we included a number of context variables: (a) maternal psychopathology (i.e., depression and anxiety); (b) family income; and (c) family composition (i.e., singlevs. dual-parent family). Finally, we included one treatment variable: therapeutic relationship, viewed by the child. Although studies have routinely included parentand child-report variables, inclusion of the teacher's view on child adjustment has been rare. Because of the scarcity of previous research, our hypotheses were tentative. We expected that greater symptom severity pretreatment, higher levels of maternal psychopathology symptoms, and fewer familial resources (e.g., lower income, single-parent family) would be associated with poor treatment response. We did not expect that sex, ethnicity, or the child's perception of the therapeutic relationship would be associated with treatment response (e.g., Kendall, 1994; Treadwell, Flannery-Schroeder, \& Kendall, 1995) but included them because of the important implications posed if they were related to treatment response. We examined predictors of response at posttreatment and 1-year follow-up separately to test for robustness of relationships.

\section{Method}

\section{Participants}

Participants ${ }^{3}$ were child clients who had completed a full course of treatment (i.e., at least 12 sessions) at

\footnotetext{
${ }^{2}$ When analyses were conducted using youth-reported diagnostic data, we found that only youth self-report of anxiety predicted treatment response. Full results are available from the first author.

${ }^{3}$ Although outcome data for $85 \%$ of the participants in this research has been examined in two separate previous articles (Kendall,
}

the Child and Adolescent Anxiety Disorders Clinic at Temple University at least 1 year prior to the current investigation. From an initial pool of 188 youth, 53 were eliminated because of incomplete data (i.e., at least one missing data point in the final pool of predictors for the posttreatment discriminant function analyses [DFAs]). Using available demographic, child-symptom (e.g., Children's Depression Inventory [CDI]), Child Behavior Checklist [CBCL], Anxiety Disorders Interview Schedule for Children [ADIS-C], Teacher's Report Form [TRF]), and parent-symptom (e.g., BDI, StateTrait Anxiety Inventory [STAI]) data, no statistically significant differences were found between those excluded $(n=53)$ and included $(n=135)$ in this study.

The final number of participants for the posttreatment analyses (hereafter, the Post sample) was 135 ( 82 boys, 53 girls; average age at intake 11.2 years, $S D=1.4$, range: 8.3 to 14.6 ). Regarding ethnicity, $86 \%$ were European American, 5\% were African American, $4 \%$ were Hispanic, $1 \%$ were Asian American, and the remaining $4 \%$ were classified as "other." The bulk of the participants $(81 \%)$ lived in a two-parent family, although a sizable minority lived with a single parent (19\%). Regarding annual family income level, $7 \%$ of the sample earned less than $\$ 20,000 ; 48 \%$ earned between $\$ 20,000$ and $\$ 49,999 ; 15 \%$ earned between $\$ 50,000$ and $\$ 69,999$; and $30 \%$ earned more than $\$ 70,000$.

Twenty-eight of the youth in the Post sample ( $n=$ 135) did not complete the 1-year follow-up assessment (i.e., $n=107$ in the follow-up sample). No statistically significant differences between those who did or did not complete the 1-year follow-up assessment were found at pretreatment or posttreatment on any demographic child-symptom (e.g., CDI, CBCL, ADIS-C, TRF) or parent-symptom (i.e., BDI, STAI) measure.

To summarize sample characteristics, the Post sample size was 135 and the follow-up sample size was 107. The Post sample includes all of the follow-up sample plus 28 additional youth who completed the posttreatment but not the 1-year follow-up assessment. As we discuss later, posttreatment analyses were conducted twice, once using the Post sample $(n=135)$ and once using the smaller follow-up sample $(n=107)$.

Youth were parent-referred to the clinic, often via an outside agency or a media advertisement. All referred children and their parents completed an informed-consent procedure and then received a full assessment, including diagnostic interview and paper-and-pencil measures. Parent-reported demographic information and parent self-report of depression and anxiety symptoms were completed via mail 1 week in advance of this

1994; Kendall et al., 1997), these analyses focus on different hypotheses and include 15\% additional "new" cases. The "new" and "old" cases did not differ on any measure at pretreatment, posttreatment, or 1-year follow-up. 
interview. After the pretreatment assessment, children who received a primary diagnosis of a Diagnostic and Statistical Manual of Mental Disorders (3rd ed., rev., or 4th ed. [DSM-III-R or DSM-IV]; American Psychiatric Association, 1987, 1994) anxiety disorder (i.e., separation anxiety disorder [SAD], generalized anxiety disorder [GAD], overanxious disorder [OAD], social phobia [SOP], or avoidant disorder [AVD]) were accepted into the study and were randomly assigned to receive treatment immediately or to be placed on an 8week waiting list. Youth were excluded if they displayed psychotic symptoms or were currently using antianxiety medications. Although both parent- and child-reported diagnoses were collected, a parent-report diagnosis was used as the primary inclusion criterion for the clinical trial (and this project). ${ }^{4}$ Only youth who were accepted into the clinical trial and completed a full course of treatment (i.e., at least 12 sessions) participated in this study.

Youth in the clinic were all treated using the same "probably efficacious" (see Ollendick \& King, 1998; Southam-Gerow \& Kendall, 2000) CBT program. The interested reader is referred to the treatment manual or the randomized clinical trial reports for a description of the treatment program (Kendall, 1994; Kendall et al., 1997; Kendall, Kane, Howard, \& Siqueland, 1990; see also Kendall, Chu, Gifford, Hayes, \& Nauta, 1998). After treatment, families were assessed two times: immediately posttreatment and 1 year later. Interviews were conducted by project staff (all clinical psychology doctoral students). It was not possible to keep interviewers naive to the time point of the interview (i.e., pretreatment, posttreatment, 1-year follow-up).

\section{Diagnostic Measures}

ADIS-Parent Version for DSM-III-R and ADIS-Parent and Child Versions for DSM-IV. The ADIS-P (Silverman, 1991) and ADIS-C (Silverman \& Albano, 1996) are structured diagnostic interviews administered to parents and children, respectively, to determine the presence of $D S M-I I I-R$ and $D S M-I V$ diagnoses in children and adolescents. Severity-impairment (i.e., "How much does the problem interfere/mess things up for you?") ratings for each diagnosis meeting criteria were also collected. A 5point scale, ranging from 0 (not at all), 1 (a little), 2 (some), 3 (a lot), to 4 (very much), was used. Supportive reliability data have been reported for the $D S M-I I I-R$ (ADIS-P: overall interrater $\kappa \quad .67$;

\footnotetext{
${ }^{4}$ Although we deviated from this practice one time in the second randomized clinical trial (see Kendall et al., 1997), we did not include that youth in this study.
}

Silverman \& Eisen, 1992; Silverman \& Rabian, 1995; ADIS-C: overall interrater $\kappa$.76; Silverman \& Eisen, 1992; Silverman \& Rabian, 1995) and the $D S M-I V$ (interrater $\kappa s$ ranging from .65 to .88 for the ADIS-P and from .57 to .80 for the ADIS-C; Silverman, Saavedra, \& Pina, in press) versions.

Interrater reliability of project diagnostic interviewers was assessed and maintained for this study in four ways. First, all new interviewers received detailed training in the use of the ADIS-C and ADIS-P, including participation in role plays and the observation of live and taped interviews conducted by experienced interviewers. Second, a training period was maintained for all new interviewers during which trainees rated tapes independently and kappa coefficients were calculated. Trainees became staff interviewers only after their kappas for all diagnoses were $\geq .80$. The third method of assuring interrater reliability involved reassessing reliability for staff interviewers on an annual basis (i.e., quality assurance). All interviewers rated tapes of interviews independently and were required to achieve kappas $\geq .80$ for all diagnoses before they could resume their interviewing duties. Although pretreatment interviews were generally used for these reliability checks (because of the greater range of diagnoses found pretreatment), posttreatment and follow-up interviews were also used to ensure that these interviews were conducted properly. Fourth, a randomly selected sample of all tapes for each interviewer was rated by the lead diagnostic interviewer, and reliability was examined. Tapes were included in the data set only if interrater reliability kappas of .80 or greater were achieved for all diagnoses. In this sample, kappas ranged from .80 to 1.00 , with a mean of .87 .

\section{Youth Report Measures}

Revised Children's Manifest Anxiety Scale. Designed to assess chronic anxiety in youth, the Revised Children's Manifest Anxiety Scale (RCMAS) self-report measure consists of 37 items to which children respond "Yes" or "No" (Reynolds \& Richmond, 1985). Psychometric data for the instrument suggest adequate reliability and validity (Reynolds \& Richmond, 1985). The measure was included to determine the relationship between self-reported childhood anxiety symptoms and treatment response.

Negative Affectivity Self-Statement Questionnaire. The Negative Affectivity Self-Statement Questionnaire (NASSQ) includes self-statements that youth endorse on a 5-point scale representing the frequency that each statement occurred in the children's thoughts during the past week (Ronan, Kendall, \& Rowe, 1994). Retest reliability, discriminative validity, concurrent 
validity, and internal consistency are all in an acceptable range (Ronan et al., 1994). The measure was included to determine the relationship between negative self-talk and treatment response.

CDI. The 27-item self-report CDI (Kovacs, 1992) is "the most widely used and researched measure of childhood depression" (Kendall, Cantwell, \& Kazdin, 1989, p. 121). In clinical samples, Cronbach's alpha for the CDI has ranged from .71 to .89 and retest reliability coefficients have ranged from .50 to .87 (see Kovacs, 1992). The measure was included to determine the relationship between childhood depressive symptoms and treatment response.

Child's Perception of Therapeutic Relationship. This 10-item questionnaire (Kendall, 1994) is completed by the youth independently after therapy (i.e., at the posttreatment interview). Children rate their perception of the quality of the youth-therapist relationship. Five items tap the child's "liking," "feeling close to," "feeling comfortable with," "talking to," and "wanting to spend more time with the therapist." Other items refer to the quality and closeness of the joint relationship. Cronbach's alpha in this sample $(n=135)$ was .74, indicating good internal reliability for the measure. The measure was included to determine the relation between the therapeutic relationship (as perceived by the child) and treatment response.

\section{Maternal Report Measures}

CBCL. The CBCL (Achenbach, 1991a) is a widely used 118-item scale that assesses parents' view of an array of behavioral problems and social competencies in their children. Available psychometric data strongly support the measure's reliability and validity (Achenbach, 1991a). As we wanted to identify specific correlates of treatment response, we chose to use the eight narrowband clinical scales (i.e., Withdrawn, Somatic Complaints, Anxious/Depressed, Social Problems, Thought Problems, Attention Problems, Delinquent Behavior, Aggressive Behavior) instead of the three broadband clinical scales. Although these narrowband scales intercorrelate moderately (e.g., from .16 to .73 in clinical samples; Achenbach, 1991a), they represent various distinct problem types. The CBCL was included to determine the relationship between maternal-reported child symptomatology and treatment response.

State-Trait Anxiety Inventory for ChildrenModification of Trait Version for Parents. Strauss (1987) modified the trait scale of the State-Trait Anxiety Inventory for Children (STAIC; Spielberger,
1973) to be used as a parent rating of youth trait anxiety. The STAIC-P-T demonstrates adequate psychometric characteristics. Southam-Gerow, Flannery-Schroeder, and Kendall (2001) reported Cronbach's alpha of .84 and retest reliability correlations of .62 for 8 weeks and .63 for 1 year. The measure also demonstrates concurrent and predictive validity. The STAIC-P-T is highly correlated (.46 to.69) with parental report of youth internalizing symptomatology (e.g., CBCL Anxious/Depressed scale), whereas correlations are in the low to medium range with (a) child-report anxiety measures (e.g., RCMAS, .09 to .33) and (b) teacher-report child internalizing symptoms (e.g., TRF Anxious/Depressed scale, .05 to .18). We included the STAIC-P-T to test the relationship between treatment response and a maternal-report measure of child anxiety.

STAI. The STAI (Spielberger, Gorusch, \& Lushene, 1970) is a widely used two-part, 40-item measure that assesses an adult's report of his or her own state and trait anxiety symptoms. Reliability, validity, and normative data support the utility of the measure and factor analyses support the state-trait distinction (e.g., Spielberger, 1973). Only the trait (STAI-T) version was used in this investigation. The measure was included to determine the relationship between maternal anxiety traits and treatment response.

BDI. A widely used 21-item scale, the BDI (Beck $\&$ Beamesderfer, 1974) assesses an adult's report of his or her own depressive symptoms. Supportive psychometric data have been amassed (Beck, Steer, \& Garbin, 1988; Kendall, Hollon, Beck, Hammen, \& Ingram, 1987). The measure was included to determine the relationship between maternal depressive symptoms and treatment response.

Demographic information. In addition, demographic information (e.g., family income, family composition) was collected from the mother.

\section{Teacher Report Measure}

TRF. The TRF (Achenbach, 1991b) parallels the parent version of the CBCL, providing a picture of the youth's classroom functioning. Achenbach (1991b) reported good psychometric characteristics for the TRF, including good retest reliability and good discriminative validity. As with the CBCL, we chose to use the eight narrowband scales instead of the three broadband scales. The TRF was included to determine the relationship between teacher-reported childhood symptomatology and treatment response. 


\section{Results}

\section{Diagnostic Profiles at Pretreatment}

Pretreatment diagnostic data for the Post sample ( $n$ $=135$ ) indicated that, according to ADIS-P (i.e., parent report), 559 of participating youth were diagnosed with primary OAD or GAD; $24 \%$ with a primary diagnosis of SAD; and $17 \%$ with a primary diagnosis of SOP or AVD. Comorbidity was extremely common in the sample; the mean number of parent-reported diagnoses was 2.7 . Only $12 \%$ of the participants met criteria for a single diagnosis. Thirty-two percent of the youth had two diagnoses, $32 \%$ met criteria for three, and $24 \%$ met criteria for four or more diagnoses. Other anxiety disorders were frequently co-occurring as secondary diagnoses, with $50 \%$ of youth meeting criteria for simple phobia, 29\% for OAD or GAD, 29\% for AVD or SOP, $15 \%$ for SAD, and $2 \%$ for obsessive-compulsive disorder. Other secondary diagnoses included $14 \%$ attention deficit hyperactivity disorder, $8 \%$ oppositional defiant disorder, $4 \%$ functional enuresis, $5 \%$ dysthymia, $4 \%$ major depressive disorder, and $2 \%$ conduct disorder.

\section{Treatment Response Categories}

The sample was divided into two groups: good treatment response versus poor treatment response, based on whether the youth met criteria for any of the intervention-targeted $D S M-I I I-R$ and $D S M-I V$ anxiety disorders (i.e., OAD, GAD, SOP, AVD, SAD) by parent report at posttreatment (and 1-year follow-up). Diagnoses were considered present if the requisite $D S M-$ $I I I-R$ or $D S M-I V$ symptoms were endorsed (using the ADIS-P) and a severity-impairment rating of at least 2 was endorsed on the 0 to 4 scale described previously. Those who did not meet criteria for any anxiety disorder (i.e., insufficient symptoms or insufficient symptom impairment) were placed in the good treatment response group, whereas those with at least one of the aforementioned anxiety disorders present at posttreatment (or 1-year follow-up) were placed in the poor treatment response group.

There were 77 youth $(57 \%)$ in the poor treatment response group at posttreatment, using the Post sample ( $n$ $=135)$; whereas for the follow-up sample $(n=107)$, there were 63 youth $(59 \%)$ classified in the poor treatment response group at posttreatment. At the 1-year follow-up point, 42 of 107 youth (39\%) were classified in the poor treatment response group. Considering stability of treatment response and focusing only on the

${ }^{5}$ Child-report diagnostic information is available from the first author. follow-up sample, we found that 74 of 107 youth (69\%) exhibited a stable treatment-response pattern (i.e., youth were classified in the same treatment-response category at both time points). Seventy-four of these, $(49 \%, n=36)$ exhibited stable poor response, and $51 \%$ $(n=38)$ exhibited stable good response. Thirty-one percent of youth $(n=33)$ exhibited an unstable treatment response. Of these "unstable responders," the vast majority ( $n=27 ; 87 \%)$ evidenced eventual good treatment response, whereas only 6 youth $(13 \%)$ evidenced a deterioration in response (i.e., from good to poor) from posttreatment to follow-up. Stated differently, $43 \%$ of youth in the poor treatment response group at posttreatment were classified as good treatment responders at 1-year follow-up, whereas only $14 \%$ of youth with a good treatment response at posttreatment were classified as poor treatment responders at 1-year follow-up.

\section{Variable Selection for Predictor Analyses}

From past research, several categories of possible predictors were considered: (a) child symptom measures, (b) parent symptom measures, (c) child characteristic measures, (d) family context measures, and (e) a therapeutic relationship measure. Accordingly, we included a broad range of child symptom measures (i.e., ADIS-P/C, CBCL, STAIC, ADIS-C, CDI, NASSQ, RCMAS, TRF) from three separate reporters ${ }^{6}$ (i.e., mother, child, teacher). In addition, we included maternal self-report measures (i.e., STAI, BDI). Several demographic variables (i.e., youth ethnicity, youth age, youth sex, family income, and family composition) were also included. All of these possible predictor variables were collected pretreatment. Finally, we included one measure of the therapeutic relationship, viewed from the child's perspective and collected at the posttreatment interview.

To reduce the pool of predictors prior to performing our classification analyses, we conducted chi-square tests and $t$ tests to examine group (i.e., poor vs. good treatment response) differences for all variables included. We retained all variables for which a statistically significant difference was found between the two treatment-response groups. Although this involved a large number of tests that typically would require the use of the Bonferroni procedure (or some similar technique to reduce Type I error), we maintained a significance level of .05 for two reasons. First, because the study was preliminary and exploratory, we felt it best to include all

\footnotetext{
${ }^{6} \mathrm{We}$ did not include father-report data because the relatively smaller sample of fathers who participated in the study $(n=91)$ would have greatly reduced our statistical power.
} 
potential predictive variables in the pool to be used in the following classification analyses. Second, we believed the more conservative method of variable selection used during the DFAs would compensate for possible concerns of this preliminary data-reduction procedure.

Chi-square tests for demographic variables (i.e., child sex, family composition [single parent, dual parent], annual family income, and child ethnicity) were conducted, comparing the treatment-response categories at both posttreatment and follow-up; all tests were nonsignificant. Means, standard deviations, test statistics, and effect-size estimates for the child symptom measures, maternal symptom measures, child age, and child's perception of the therapeutic relationship are presented in Tables 1 and 2. For the posttreatment tests of differences between the two treatment response groups, effect sizes for maternal report of child symptoms ranged from small to medium (.07 to .55), child self-report effect sizes were all small (.01 to .14), and teacher report of child symptom-effect sizes were small to medium (.06 to .38). At follow-up, maternal report of child symptom-effect sizes ranged from small to medium (.04 to .72), child self-report effect sizes were all small (.04 to .25$)$, and teacher report of child symptomeffect sizes ranged from small to medium (.07 to .57). Maternal self-report effect sizes were medium (.37) at

Table 1. Descriptive and Test Statistics of Participant Measures: Treatment Response Posttreatment

\begin{tabular}{|c|c|c|c|c|c|c|c|}
\hline & \multicolumn{2}{|c|}{ Poor Response } & \multicolumn{2}{|c|}{ Good Response } & \multirow[b]{2}{*}{$t$} & \multirow[b]{2}{*}{$d f$} & \multirow[b]{2}{*}{ Cohen's $d$} \\
\hline & $M$ & $S D$ & $M$ & $S D$ & & & \\
\hline \multicolumn{8}{|l|}{ Demographic } \\
\hline Child Age (in Months) & 137.22 & 17.95 & 130.00 & 13.81 & $2.55^{*}$ & 133 & 0.44 \\
\hline \multicolumn{8}{|c|}{ Parent Report ( $T$ Scores Unless Indicated) } \\
\hline No. Parent-Report dx $\mathrm{c}$ & 2.78 & 1.25 & 2.69 & 0.96 & 0.45 & 133 & 0.08 \\
\hline CBCL-1 & 67.78 & 8.95 & 62.57 & 9.98 & $3.19 *$ & 133 & 0.55 \\
\hline CBCL-2 & 65.81 & 10.43 & 67.21 & 9.77 & -0.79 & 133 & 0.14 \\
\hline CBCL-3 & 72.84 & 9.09 & 70.29 & 9.98 & 1.55 & 133 & 0.27 \\
\hline CBCL-4 & 65.03 & 11.59 & 61.79 & 9.39 & 1.74 & 133 & 0.30 \\
\hline CBCL-5 & 59.70 & 8.07 & 60.79 & 9.31 & -0.73 & 133 & 0.13 \\
\hline CBCL-6 & 63.00 & 9.23 & 61.41 & 9.37 & 0.98 & 133 & 0.17 \\
\hline CBCL-7 & 53.43 & 5.45 & 53.19 & 4.77 & 0.27 & 133 & 0.05 \\
\hline CBCL-8 & 55.77 & 6.70 & 54.76 & 6.57 & 0.87 & 133 & 0.15 \\
\hline STAIC-P-Ta & 54.13 & 8.00 & 53.71 & 7.62 & 0.30 & 130 & 0.05 \\
\hline BDIa & 41.01 & 9.72 & 37.43 & 9.23 & $2.18^{*}$ & 133 & 0.37 \\
\hline STAI-Ta & 41.01 & 9.72 & 37.43 & 9.23 & $2.17^{*}$ & 133 & 0.37 \\
\hline \multicolumn{8}{|l|}{ Teacher-Report ( $T$ Scores) } \\
\hline TRF-1 & 62.71 & 11.18 & 59.52 & 9.00 & 1.79 & 133 & 0.31 \\
\hline TRF-2 & 58.12 & 10.35 & 56.66 & 9.06 & 0.86 & 133 & 0.15 \\
\hline TRF-3 & 65.77 & 10.95 & 62.19 & 9.23 & $2.01 *$ & 133 & 0.35 \\
\hline TRF-4 & 60.06 & 8.72 & 57.36 & 8.10 & 1.84 & 133 & 0.32 \\
\hline TRF-5 & 58.06 & 9.36 & 59.66 & 9.31 & -0.98 & 133 & 0.17 \\
\hline TRF-6 & 55.97 & 7.36 & 55.43 & 6.62 & 0.44 & 133 & 0.08 \\
\hline TRF-7 & 52.55 & 4.10 & 52.45 & 4.46 & 0.13 & 133 & 0.02 \\
\hline TRF-8 & 54.05 & 5.88 & 53.22 & 5.04 & 0.86 & 133 & 0.15 \\
\hline \multicolumn{8}{|c|}{ Youth-Report (Raw Scores Unless Indicated) } \\
\hline No. Child-Report $\mathrm{dx}^{\mathrm{c}}$ & 1.86 & 1.32 & 1.53 & 1.30 & 1.42 & 133 & 0.24 \\
\hline RCMASb & 54.56 & 10.85 & 53.74 & 9.48 & 0.46 & 133 & 0.08 \\
\hline NASSQ & 61.64 & 27.88 & 61.88 & 25.23 & -0.05 & 132 & 0.01 \\
\hline STAIC-T & 52.92 & 12.97 & 51.00 & 12.79 & 0.86 & 133 & 0.15 \\
\hline CDI & 11.43 & 7.81 & 10.64 & 8.41 & 0.57 & 132 & 0.10 \\
\hline CPTR & 23.12 & 5.39 & 23.78 & 5.16 & -0.67 & 115 & 0.12 \\
\hline
\end{tabular}

Note: All scores reported were from the measures collected pretreatment except the CPTR. CBCL-1 = Child Behavior Checklist-Withdrawn subscale; CBCL-2 = Child Behavior Checklist-Somatic Problems subscale; CBCL-3 = Child Behavior Checklist-Anxious/Depressed subscale; CBCL-4 = Child Behavior Checklist-Social Problems subscale; CBCL-5 = Child Behavior Checklist-Thought Problems subscale; CBCL-6 = Child Behavior Checklist-Attention Problems subscale; CBCL-7 = Child Behavior Checklist-Delinquent Behavior subscale; CBCL-8 = Child Behavior Checklist-Aggressive Behavior subscale; STAIC-P-Trait $=$ State-Trait Anxiety Inventory for Children-Parent version; BDI $=$ Beck Depression Inventory; STAI-T: State-Trait Anxiety Inventory-Trait version; TRF-1 = Teacher Report Form, Withdrawn subscale; TRF-2 = Teacher Report Form, Somatic Problems subscale TRF-3 = Teacher Report Form, Anxious/Depressed subscale; TRF $-4=$ Teacher Report Form, Social Problems subscale; TRF-5 = Teacher Report Form, Thought Problems subscale; TRF-6 = Teacher Report Form, Attention Problems subscale TRF-7 = Teacher Report Form, Delinquent Behavior subscale; TRF-8 = Teacher Report Form, Aggressive Behavior subscale; RCMAS $=$ Revised Clinical Manifest Anxiety Scale-Anxiety scale; NASSQ = Negative Affectivity Self-Statement Questionnaire; STAIC - T $=$ State Trait Anxiety Inventory for Children-Trait version; CDI = Children's Depression Inventory; CPTR = Child's Perception of Therapeutic Relationship.

${ }^{\mathrm{a} R a w}$ scores reported. ${ }^{\mathrm{b}} T$ scores reported. ${ }^{\mathrm{c}}$ Frequencies reported. $* p<.05$. 
CORRELATES OF TREATMENT RESPONSE

Table 2. Descriptive and Test Statistics of Participant Measures: Treatment Response 1-Year Follow-Up

\begin{tabular}{|c|c|c|c|c|c|c|c|}
\hline & \multicolumn{2}{|c|}{ Poor Response } & \multicolumn{2}{|c|}{ Good Response } & \multirow[b]{2}{*}{$\mathbf{t}$} & \multirow[b]{2}{*}{$d f$} & \multirow[b]{2}{*}{ Cohen's $d$} \\
\hline & $M$ & $S D$ & $M$ & $S D$ & & & \\
\hline \multicolumn{8}{|l|}{ Demographic } \\
\hline Child Age (in Months) & 134.19 & 16.75 & 133.34 & 16.67 & 0.26 & 105 & 0.05 \\
\hline \multicolumn{8}{|c|}{ Parent-Report ( $T$ Scores Unless Indicated) } \\
\hline No. Parent-Report dxc & 2.83 & 1.10 & 2.69 & 1.18 & 0.62 & 105 & 0.12 \\
\hline CBCL-1 & 69.79 & 8.59 & 63.68 & 9.53 & $3.36^{*}$ & 105 & 0.66 \\
\hline CBCL-2 & 65.67 & 10.64 & 66.55 & 10.40 & -0.43 & 105 & 0.08 \\
\hline CBCL-3 & 74.79 & 8.63 & 70.71 & 10.37 & $2.12 *$ & 105 & 0.42 \\
\hline CBCL-4 & 65.83 & 12.19 & 63.00 & 9.88 & 1.32 & 105 & 0.26 \\
\hline CBCL-5 & 60.14 & 7.91 & 59.35 & 9.32 & 0.45 & 105 & 0.09 \\
\hline CBCL-6 & 64.38 & 9.70 & 61.14 & 9.17 & 1.75 & 105 & 0.34 \\
\hline CBCL-7 & 54.21 & 5.86 & 53.11 & 4.95 & 1.05 & 105 & 0.20 \\
\hline CBCL-8 & 56.24 & 7.19 & 55.08 & 6.95 & 0.83 & 105 & 0.16 \\
\hline STAIC-P-Ta & 55.05 & 6.27 & 53.28 & 8.70 & 1.11 & 99 & 0.22 \\
\hline BDIa & 11.24 & 9.66 & 6.88 & 4.70 & $3.12 *$ & 105 & 0.61 \\
\hline STAI-Ta & 41.98 & 10.55 & 37.92 & 9.00 & $2.13^{*}$ & 105 & 0.42 \\
\hline \multicolumn{8}{|l|}{ Teacher-Report ( $T$ Scores) } \\
\hline TRF-1 & 63.98 & 11.32 & 61.08 & 10.22 & 1.37 & 105 & 0.27 \\
\hline TRF-2 & 59.14 & 10.77 & 57.05 & 9.67 & 1.05 & 105 & 0.20 \\
\hline TRF-3 & 68.00 & 10.58 & 62.40 & 9.58 & $2.83^{*}$ & 105 & 0.56 \\
\hline TRF-4 & 62.12 & 9.40 & 57.12 & 6.92 & $3.10^{*}$ & 105 & 0.62 \\
\hline TRF-5 & 59.64 & 9.76 & 57.55 & 9.04 & 1.13 & 105 & 0.22 \\
\hline TRF-6 & 56.98 & 8.15 & 55.71 & 6.85 & 0.87 & 105 & 0.17 \\
\hline TRF-7 & 52.79 & 4.30 & 52.58 & 4.59 & 0.23 & 105 & 0.05 \\
\hline TRF-8 & 55.38 & 6.24 & 52.98 & 4.85 & $2.23^{*}$ & 105 & 0.44 \\
\hline \multicolumn{8}{|c|}{ Youth-Report (Raw Scores Unless Indicated) } \\
\hline No. Child-Report $\mathrm{dx}^{\mathrm{c}}$ & 1.98 & 1.26 & 1.63 & 1.38 & 1.31 & 105 & 0.26 \\
\hline RCMASb & 55.26 & 12.25 & 53.34 & 8.95 & 0.94 & 105 & 0.18 \\
\hline NASSQ & 65.88 & 34.75 & 62.54 & 21.40 & 0.61 & 104 & 0.12 \\
\hline STAIC-T & 53.05 & 13.35 & 51.29 & 13.00 & 0.68 & 105 & 0.13 \\
\hline CDI & 12.85 & 9.46 & 10.63 & 7.63 & 1.33 & 104 & 0.26 \\
\hline CPTR & 23.08 & 4.89 & 23.72 & 5.53 & -0.56 & 87 & 0.12 \\
\hline
\end{tabular}

Note: All scores reported were from the measures collected pretreatment except the CPTR. CBCL-1 = Child Behavior Checklist-Withdrawn subscale; CBCL-2 = Child Behavior Checklist-Somatic Problems subscale; CBCL-3 = Child Behavior Checklist-Anxious/Depressed subscale; CBCL-4 = Child Behavior Checklist-Social Problems subscale; CBCL-5 = Child Behavior Checklist-Thought Problems subscale; CBCL-6 = Child Behavior Checklist-Attention Problems subscale; CBCL-7 = Child Behavior Checklist-Delinquent Behavior subscale; CBCL-8 = Child Behavior Checklist-Aggressive Behavior subscale; STAIC-P-Trait $=$ State-Trait Anxiety Inventory for Children-Parent version; BDI = Beck Depression Inventory; STAI-T = State-Trait Anxiety Inventory-trait version; TRF-1 = Teacher Report Form, Withdrawn subscale; TRF-2 = Teacher Report Form, Somatic Problems subscale TRF-3 = Teacher Report Form, Anxious/Depressed subscale; TRF-4 = Teacher Report Form, Social Problems subscale; TRF-5 = Teacher Report Form, Thought Problems subscale; TRF-6 = Teacher Report Form, Attention Problems subscale TRF-7 = Teacher Report Form, Delinquent Behavior subscale; TRF-8 = Teacher Report Form, Aggressive Behavior subscale; RCMAS $=$ Revised Clinical Manifest Anxiety Scale-Anxiety scale; NASSQ = Negative Affectivity Self-Statement Questionnaire; STAIC-T = StateTrait Anxiety Inventory for Children-Trait version; CDI = Children's Depression Inventory; CPTR = Child's Perception of Therapeutic Relationship.

${ }^{a}$ Raw scores reported. ${ }^{b} T$ scores reported. ${ }^{\mathrm{c}}$ Frequencies reported. $* p<.05$.

posttreatment and medium (.42 to .61) at follow-up. The effect size for child age was medium at posttreatment (.47) and small at follow-up (.10). Finally, the effect size for the treatment relationship variable was small at both the posttreatment (.12) and 1-year follow-up (.12) assessment point.

All variables for which a significant difference was found between the treatment response categories were retained for the DFA. Thus, for the posttreatment analysis, the following variables were included: child age, maternal BDI, maternal STAI trait, CBCL Withdrawn subscale, and TRF Anxious/Depressed subscale. For the 1-year follow-up analysis, the following variables were included: maternal BDI, maternal STAI trait, CBCL Withdrawn subscale, CBCL Anxious/Depressed subscale, TRF Anxious/Depressed subscale, TRF Social Problems subscale, and TRF Aggressive Behavior subscale.

\section{DFA}

Two DFAs were conducted - one for treatment response at posttreatment and one for treatment response at follow-up. Both analyses were conducted stepwise, 
in order of the reduction of Wilks's lambda. Preliminarily, no threat to these multivariate analyses was indicated when assumptions were evaluated. Table 3 provides the results of the two analyses. For treatment response at posttreatment $(n=135)$, three variables remained in the equation, entered in the following order: (a) CBCL Withdrawn narrowband scale, (b) youth age, and (c) TRF Anxious/Depressed narrowband scale. Higher scores on the CBCL Withdrawn narrowband scale and TRF Anxious/Depressed narrowband scale and older-youth age were associated with poor treatment response. A second DFA was conducted for treatment response at posttreatment, including only youth in the smaller follow-up sample $(n=107)$. Results, reported in Table 3, were consistent with those found with the larger Post sample $(n=135)$. The same three variables remained in the equation, entered in the same order (i.e., CBCL Withdrawn narrowband scale, youth age, and TRF Anxious/Depressed narrowband scale).

Using a jacknifed classification, for treatment response at 1-year follow-up $(n=107)$, three variables remained in the discriminant equation, entered in the following order: (a) CBCL Withdrawn narrowband scale, (b) maternal BDI, and (c) TRF Anxious/Depressed narrowband scale. Higher scores on the CBCL Withdrawn narrowband scale, TRF Anxious/Depressed narrowband scale, and maternal BDI were associated with poor treatment response. Using a jackknifed classification procedure, we found that the three functions correctly classified $68 \%, 71 \%$, and $72 \%$ of participants. Using the procedure recommended by Tabachnick and Fidell (1996), these statistics were both considerably greater than would be expected by chance when correcting for prior probabilities and considering the unequal sample sizes $(51 \%, 52 \%$, and $52 \%$, respectively).

\section{Discussion}

This investigation identified correlates of treatment response in a clinic using individual CBT for children with anxiety disorders. Treatment response for children who received a full course of treatment was evaluated for parent report of child diagnoses (poor response $=$ any of five $D S M-I I I-R$ and $D S M-I V$ anxiety disorders still present after treatment), separately, at two assessment points (posttreatment and 1-year follow-up) using DFA. A number of treatment-response predictors emerged, most of which were consistent with past research. Poor treatment response was predicted by (a) higher levels of specific youth symptoms (e.g., withdrawal, anxiety or depression), as reported by two sources (i.e., mother and teacher); (b) high levels of ma- ternal depressive symptoms (at follow-up only); and (c) older youth age (at posttreatment only). Many potential correlates of parent-reported treatment response, such as maternal- and teacher-reported externalizing symptoms (e.g., delinquent behavior, attention problems), youth-reported symptoms (e.g., depressive symptoms, anxiety symptoms), number of comorbid disorders, and several demographic variables (i.e., ethnicity, family income, family composition, and sex) were not significantly related to treatment response according to the DFA. Overall, the discriminant functions classified youth much better than would be expected by chance, and the classification statistics were comparable with the few other studies of treatment response (e.g., Clarke et al., 1992; Webster-Stratton, 1985).

On a positive note, response to a full course of a CBT program in this sample was not predicted by demographic factors such as family income, family composition, or sex. In addition, level of noninternalizing youth psychopathology symptoms (e.g., number of comorbid disorders, CBCL, and TRF scales, such as delinquent behavior and attention problems) also did not predict treatment response in our classification analyses. These findings offer some support for the contention that the treatment may be transportable to diverse clinical contexts (Southam-Gerow \& Kendall, 2000; see also Kendall \& Southam-Gerow, 1995). However, because there was relatively limited variability of externalizing behavior problems in the children treated at our clinic, this finding should be interpreted cautiously. Indeed, in different clinical settings where children experience higher levels of externalizing behavior problems, treatment response may not be as positive (for discussion, see Weisz, 2000).

One of the correlates associated with poor treatment response was higher levels of internalizing problems as reported by two separate reporters, indexed by the CBCL Withdrawn scale (sample items: "Rather be alone," "Shy," "Sulks,") and the TRF Anxious/Depressed scale (sample items: "Cries," "Fearful," "Worries"). Severity of symptoms pretreatment has been a consistent predictor of poor treatment response for CBT across several studies involving youth with depressive disorders (e.g., Brent et al., 1998; Clarke et al., 1992; Jayson et al., 1998), although methods in those studies differed from the methods of this study (e.g., child report of symptoms vs. parent report of symptoms predicting response). These results are quite robust because the findings were uniform across two time points and two reporters. Possible adaptations to the CBT program are suggested by this finding. For example, the "dose" of treatment may need to be increased (e.g., more sessions, booster sessions) for some youth. In addition, inclusion of specific adjunctive-treatment com- 


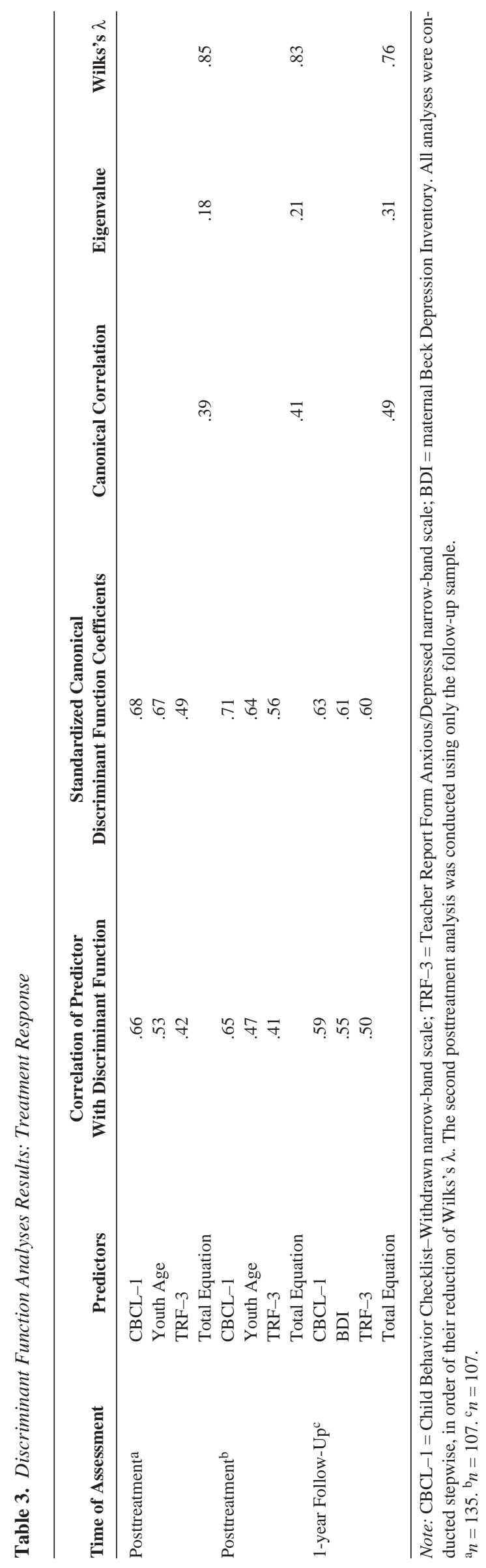


ponents that address the moderators of treatment response may help. For instance, social skills training (e.g., Albano \& Barlow, 1996; Beidel \& Turner, 1998) or activity-selection interventions (e.g., Lewinsohn, Clarke, Rohde, \& Hops, 1996) may address the specific symptoms (e.g., social withdrawal) that predicted a poor treatment response in this study.

Mothers of youth classified in the poor treatment-response group at follow-up reported high levels of selfreported depressive symptoms at the intake. The relation of maternal psychopathology symptoms with poorer outcome synergizes with current thought about the role of the family (particularly parents) in childhood anxiety disorders (e.g., Chorpita \& Barlow, 1998; Ginsburg, Silverman, \& Kurtines, 1995) and is consistent with past work on treatment response in conductdisordered youth (e.g., Patterson \& Chamberlain, 1994). In addition, research on emotional development suggests that children of depressed mothers are at high risk for poor outcomes, particularly emotion-regulation problems (e.g., Field, Pickens, Fox, Nawrocki, \& Gonzalez, 1995; Goodman, Brogan, Lynch, \& Fielding, 1993; Zahn-Waxler, Kochanska, Krupnick, \& McKnew, 1990; for review, see Southam-Gerow \& Kendall, in press). Thus, the mechanism behind the poorer treatment response for these children may be linked to parent-child relational factors, either longstanding (e.g., delayed emotional development) or more proximal (e.g., depressed mothers may be less able to ensure child treatment compliance). The finding suggests that the integration of a family component to treatment may also be helpful to some youth. Family CBT programs have been quite successful at helping anxietydisordered youth (e.g., Barrett, Dadds, \& Rapee, 1996; Cobham, Dadds, \& Spence, 1998; Howard \& Kendall, 1996; Silverman et al., 1999). The Cobham et al. study is particularly noteworthy because they found that the superiority of family CBT to individual CBT was most pronounced when the level of maternal anxiety symptomatology was high. Adjunctive individual therapy for the parent may also prove helpful. Thus, increased involvement of parents in treatment appears a reasonable approach to enhance outcomes.

Older age was associated with less favorable treatment response at posttreatment. There were a priori reasons to predict that increased age would have a positive (e.g., older youth are better able to take advantage of cognitive treatments; see Durlak et al., 1991; Weisz \& Weersing, 1998) or a negative (e.g., Jayson et al., 1998; Patterson \& Chamberlain, 1994) impact on outcomes. Because initial severity of psychopathology was not correlated with age in our sample, we can only speculate about why older age was associated with poorer treatment response. It may be that older youth have a more chronic, intractable disorder, the extent of which was not adequately tapped by our measurement (e.g., duration of symptoms was not collected). In addi- tion, it is possible that highly anxious older youth may be more "non-normative," developmentally speaking, and the problems associated with these disorders may cause more interference for older youth as they navigate the challenges associated with adolescence (e.g., increased autonomy). It is also conceivable that the program is less agreeable or palatable to older youth, who may view some of the exercises and assignments as somewhat "childish."7 A more speculative possibility is that a variant of the "early starter" model (e.g., Patterson, Capaldi, \& Bank, 1991; see also Hinshaw, Lahey, \& Hart, 1993; Loeber, 1988; Moffitt, 1993) described in the literature on antisocial youth may have some application with anxiety disorders. For example, there may have been a higher proportion of older youth in our sample whose anxiety disorders arose from a combination of inhibited temperament (e.g., see Biederman et al., 1993; Kagan, Reznick, \& Snidman, 1988), early autonomy-constraining family environment (see Chorpita \& Barlow, 1998; Ginsburg et al., 1995), and delayed or constricted emotional development (emotion dysregulation; e.g., Keenan, 2000; Southam-Gerow \& Kendall, in press). "Late-starter" anxious youth may have developed the disorder less from temperamental, familial, and emotion-regulation risk factors and more from uncontrollable, acute anxiety-producing environmental events (e.g., marital conflict, parental illness). Because this project did not assess these possibilities, future work will benefit from their consideration.

Because older youth fared less well, some modifications to a CBT program may augment outcomes for them. First, more intensive treatment for older youth may be indicated (e.g., longer course of treatment). Second, for older youth the treatment may need modification to be more "teen-friendly" (e.g., use a college notebook instead of the standard homework book, reduce didactic nature of some sessions). Finally, there may be a need for early intervention and prevention as a more proactive method for alleviating anxiety disorders in youth (e.g., Dadds et al., 1999; Dadds, Spence, Holland, \& Barrett, 1997).

The absence of an effect for the treatment relationship deserves some discussion. The adult literature suggests the importance of therapeutic relationship to treatment outcome (e.g., Horvath \& Luborsky, 1993; Orlinsky \& Howard, 1986). Similarly, children and families have often reported the experience of a positive therapeutic relationship as the most important dimension of treatment (e.g., Kendall \& SouthamGerow, 1996; Motta \& Lynch, 1990). However, although the importance of the therapeutic relationship to child clients (and their families) seems clear, the actual effects of the relationship on outcome are less well es-

\footnotetext{
${ }^{7}$ Thanks to Bruce Compas for suggesting this possibility.
} 
tablished. For behavioral and cognitive-behavioral treatments, parents and children have rated the relationship as central to change, but actual correlations of therapeutic relationship quality with positive outcome, although in the correct directions, were generally nonsignificant (Kendall, 1994; Motta \& Lynch, 1990; Motta \& Tobin, 1992; but see Eltz, Shirk, \& Sarlin, 1995; Shirk, Saiz, \& Sarlin, 1993, for data on the importance of the treatment relationship in nonbehavioral therapies). Although our results are in line with previous null findings on the effect of the therapeutic relationship in CBT, there are a few factors of note that may have impacted our findings. First, child ratings of treatment-relationship quality were near the top of the scale for the measure; thus correlations with outcome may have been obscured by ceiling effects. Relatedly, had we included youth who dropped out of treatment prematurely, it is conceivable that different results would have been found for the treatment-relationship variable. Finally, we used only a single child-report measure of the relationship (and at only one time point), the validity of which is unknown. It is our view that the relationship is important but that these factors reduced variability and precluded predictive relationships.

A few closing comments about judging poor and good treatment response are in order. First, we remind the reader that we chose quite stringent criteria in defining the treatment-response categories. Thus, a child with four of the five target anxiety-disorder diagnoses at pretreatment and only one at posttreatment would still be categorized as a "poor" responder. We chose this conservative method because it paralleled past research and because we were interested in predictors of optimal versus nonoptimal outcomes to inform further treatment development. However, we emphasize our hesitation about using terms such as poor treatment response. Change and response in therapy are complex processes, and our measurement, although typical, remains imperfect. A child categorized as a poor treatment responder, as indexed by our measures, may believe he or she has experienced substantial improvement in his or her life in ways that we do not or cannot assess. Although our categorizations have empirical grounding, "false positives" remain a possibility. As Kendall (1989) argued, some therapy research has placed an unfortunate emphasis on "cure" rates. It may be irrational to expect an absence of psychopathology after a course of psychotherapy. Instead, an increasing sense of ability to manage one's psychological life may be a more reasonable and attainable goal (Kendall, 1989; see also Southam-Gerow \& Kendall, 2000).

This project was not without its shortcomings. The classification statistics, although better than would be expected by chance and comparable to those achieved by other investigators, were only in the moderate range. Second, the function was not cross-validated with a second sample. In addition, the study could have been improved by a wider range of both measurements of child psychopathology and other possible factors associated with treatment response (e.g., paternal psychopathology, additional treatment-relationship variables). Relatedly, although we assessed a wide variety of indexes of child functioning, some areas were not measured (e.g., academic functioning, social relations). In addition, the low participation of fathers in the assessments of our sample is a concern (e.g., Phares, 1996; Phares \& Compas, 1992). Furthermore, other nonspecific factors, such as client engagement, therapist warmth, and therapist-parent cohesion may have contributed to treatment response. Again, we did not assess these dimensions and thus cannot draw conclusions about their relative importance. Another potential caveat regarding our pool of predictors was that our sample did not include a full representation of several ethnic groups to adequately test whether ethnicity was related to treatment response. Finally, our sample size, although relatively large, did not offer power to examine complex interactions between important predictors. Future work with larger samples will need to address such an important possibility.

Finally, there are two additional issues for readers to consider when integrating these findings into the literature. First, in addition to the categorical divisions used in this study, we considered examining treatment response, using a dimensional model of psychopathology (e.g., examining CBCL scores; cf. Clarke et al., 1992). Although a dimensional model has merit, in this instance, we chose a categorical approach for several reasons. First, diagnostic data were used as the sole mode of entry into the clinic. Although categorical and dimensional data tend to correlate, no effort was made to assure this in the sample. With diagnostic data, the starting point for all participants would be relatively equivalent (i.e., at least one anxiety-disorder diagnosis) whereas for continuous measures, there would be no such uniform starting point. Second, a categorical approach is consistent with most other research in the area (e.g., Brent et al., 1998; Jayson et al., 1998). Third, using diagnostic status as an indicator of treatment response is in line with calls to focus on clinically significant outcomes in psychotherapy research (e.g., Jacobson, Roberts, Berns, \& McGlinchey, 1999). However, using a dimensional model may have yielded different results (see Clarke et al., 1992).

Our sole reliance on parent report to index treatment response is another important issue to take into account when considering these findings. Although we had good rationale for focusing our project in this way (e.g., parent-report diagnosis was our primary inclusion criterion; parents referred all children to the clinic), our own exploratory analyses indicated that using child report yielded different findings (e.g., only child-report variables predicted treatment response). This result is not surprising given past research documenting that 
parent and child reports on child symptoms and diagnoses are generally discrepant (e.g., Achenbach, McConaughy, \& Howell, 1987; Garber, Van Slyke, \& Walker, 1998; Rapee, Barrett, Dadds, \& Evans, 1994). The choice to emphasize parent report has important consequences and provides only part of the picture. Still, we believe our findings provide an important glimpse into the predictors of treatment response for children with anxiety disorders.

\section{References}

Achenbach, T. M. (1991a). Manual for the Child Behavior Checklists/4-18 and 1991 profile. Burlington: University of Vermont.

Achenbach, T. M. (1991b). Manual for the Teacher Report Form and 1991 Profile. Burlington: University of Vermont, Department of Psychiatry.

Achenbach, T. M., McConaughy, S. H., \& Howell, C. T. (1987). Child/adolescent behavioral and emotional problems: Implications of cross-informant correlations for situational specificity. Psychological Bulletin, 101, 213-232.

Albano, A. M., \& Barlow, D. H. (1996). Breaking the vicious cycle: Cognitive-behavioral group treatment for socially anxious youth. In E. D. Hibbs \& P. S. Jensen (Eds.), Psychosocial treatments for child and adolescent disorders: Empirically based strategies for clinical practice (pp. 43-62). Washington, DC: American Psychological Association.

American Psychiatric Association. (1987). Diagnostic and statistical manual of mental disorders (3rd ed., rev.). Washington, DC: Author.

American Psychiatric Association. (1994). Diagnostic and statistical manual of mental disorders (4th ed.). Washington, DC: Author.

Armbruster, P., \& Kazdin, A. E. (1994). Attrition in child psychotherapy. Advances in Clinical Child Psychology, 16, 81-108.

Barrett, P. M., Dadds, M. R., \& Rapee, R. M. (1996). Family treatment of childhood anxiety: A controlled trial. Journal of Consulting and Clinical Psychology, 64, 333-342.

Beck, A. T., \& Beamesderfer, A. (1974). Assessment of depression: The Depression Inventory. In P. Pichot (Ed.), Psychological measurement in psychopharmacology (pp. 151-169). Paris: Karger.

Beck, A. T., Steer, R. A., \& Garbin, M. G. (1988). Psychometric properties of the Beck Depression Inventory: Twenty-five years of evaluation. Clinical Psychology Review, 8, 77-100.

Beidel, D. C., \& Turner, S. M. (1998). Shy children, phobic adults: Nature and treatment of social phobia. Washington, DC: American Psychological Association.

Biederman, J., Rosenbaum, J. F., Bolduc-Murphy, E. A., Faraone, S. V., Chaloff, J., Hirshfeld, D. R., \& Kagan, J. (1993). Behavioral inhibition as a temperamental risk factor for anxiety disorders. Child and Adolescent Psychiatric Clinics of North America, 2, 667-684.

Brent, D. A., Kolko, D. J., Birmaher, B., Baugher, M., Bridge, J., Roth, C., \& Holder, D. (1998). Predictors of treatment efficacy in a clinical trial of three psychosocial treatments for adolescent depression. Journal of the American Academy of Child and Adolescent Psychiatry, 37, 906-914.

Bush, R. M., Glenwick, D. S., \& Stephens, M. A. P. (1986). Predictors of psychotherapy outcome for children at a community mental health center. Journal of Clinical Psychology, 42, 873877.

Casey, R. J., \& Berman, J. S. (1985). The outcome of psychotherapy with children. Psychological Bulletin, 98, 388-400.
Chorpita, B. F., \& Barlow, D. H. (1998). The development of anxiety: The role of control in the early environment. Psychological Bulletin, 124, 3-21

Clarke, G., Hops, H., Lewinsohn, P. M., Andrews, J., Seeley, J. R., \& Williams, J. (1992). Cognitive-behavioral group treatment of adolescent depression: Prediction of outcome. Behavior Therapy, 23, 341-354.

Cobham, V. E., Dadds, M. R., \& Spence, S. H. (1998). The role of parental anxiety in the treatment of childhood anxiety. Journal of Consulting and Clinical Psychology, 66, 893-905.

Dadds, M. R., Holland, D. E., Laurens, K. R., Mullins, M., Barrett, P. M., \& Spence, S. H. (1999). Early intervention and prevention of anxiety disorders in children: Results at 2-year follow-up. Journal of Consulting and Clinical Psychology, 67, 145-150.

Dadds, M. R., Schwartz, S., \& Sanders, M. R. (1987). Marital discord and treatment outcome in behavioral treatment of child conduct disorders. Journal of Consulting and Clinical Psychology, 55, 396-403.

Dadds, M. R., Spence, S. H., Holland, D. E., \& Barrett, P. M. (1997). Prevention and early intervention for anxiety disorders: A controlled trial. Journal of Consulting and Clinical Psychology, 65, $627-635$.

Dumas, J. E. (1984). Interactional outcomes of treatment outcome in behavioral parent training. Journal of Consulting and Clinical Psychology, 52, 946-954.

Dumas, J. E., \& Wahler, R. G. (1983). Predictors of outcome in parent training: Mother insularity and socioeconomic disadvantage. Behavioral Assessment, 5, 301-313.

Durlak, J. A., Fuhrman, T., \& Lampman, C. (1991). Effectiveness of cognitive-behavior therapy for maladapting children: A metaanalysis. Psychological Bulletin, 110, 204-214.

Eltz, M. J., Shirk, S. R., \& Sarlin, N. (1995). Alliance formation and treatment outcome among maltreated adolescents. Child Abuse and Neglect, 19, 419-431.

Field, T., Pickens, J., Fox, N. A., Nawrocki, T., \& Gonzalez, J. (1995). Vagal tone in infants of depressed mothers. Development and Psychopathology, 7, 227-231.

Frick, P. J., Silverthorn, P., \& Evans, C. (1994). Assessment of childhood anxiety using structured interviews: Patterns of agreement among informants and association with maternal anxiety. Psychological Assessment, 6, 372-379.

Garber, J., Van Slyke, D. A., \& Walker, L. S. (1998). Concordance between mothers' and children's reports of somatic and emotional symptoms in patients with recurrent abdominal pain or emotional disorders. Journal of Abnormal Child Psychology, 26, 381-391.

Ginsburg, G. S., Silverman, W. K., \& Kurtines, W. K. (1995). Family involvement in treating children with phobic and anxiety disorders: A look ahead. Clinical Psychology Review, 15, 457-473.

Goodman, S. H., Brogan, D., Lynch, M. E., Fielding, B. (1993). Social and emotional competence in children of depressed mothers. Child Development, 64, 516-531.

Henggeler, S. W., Melton, G. B., Brondino, M. J., Scherer, D. G., \& Hanley, J. H. (1997). Multisystemic therapy with violent and chronic juvenile offenders and their families: The role of treatment fidelity in successful dissemination. Journal of Consulting and Clinical Psychology, 65, 821-833.

Hinshaw, S. P., Lahey, B. B., \& Hart, E. L. (1993). Issues of taxonomy and comorbidity in the development of conduct disorder. Development and Psychopathology, 5, 31-49.

Horvath, A. O., \& Luborsky, L. (1993). The role of the therapeutic alliance in psychotherapy. Journal of Consulting and Clinical Psychology, 61, 561-573.

Howard, B. L., \& Kendall, P. C. (1996). Cognitive-behavioral family therapy for anxiety-disordered children: A multiple-baseline evaluation. Cognitive Therapy \& Research, 20, 423-443.

Jacobson, N. S., Roberts, L. J., Berns, S. B., \& McGlinchey, J. B. (1999). Methods for defining and determining the clinical sig- 
nificance of treatment effects: Description, application, and alternatives. Journal of Consulting \& Clinical Psychology, 67, 300-307.

Jayson, D., Wood, A., Kroll, L., Fraser, J., \& Harrington, R. (1998). Which depressed patients respond to cognitive-behavioral treatment. Journal of the American Academy of Child and Adolescent Psychiatry, 37, 906-914.

Kagan, J., Reznick, J. S., \& Snidman, N. (1988). Biological bases of childhood shyness. Science, 240, 167-171.

Kazdin, A. E., Bass, D., Ayers, W. A., \& Rodgers, A. (1990). Empirical and clinical focus of child and adolescent psychotherapy research. Journal of Consulting \& Clinical Psychology, 58, 729740.

Keenan, K. (2000). Emotion dysregulation as a risk factor for child psychopathology. Clinical Psychology: Science and Practice, 7, 418-434.

Kendall, P. C. (1989). The generalization and maintenance of behavior change: Comments, considerations, and the "no-cure" criticism. Behavior Therapy, 20, 357-364.

Kendall, P. C. (1994). Treating anxiety disorders in youth: Results of a randomized clinical trial. Journal of Consulting and Clinical Psychology, 62, 100-110.

Kendall, P. C., Cantwell, D. P., \& Kazdin, A. E. (1989). Depression in children and adolescents: Assessment issues and recommendations. Cognitive Therapy and Research, 13, 109-146.

Kendall, P. C., Chu, B., Gifford, A., Hayes, C., \& Nauta, M. (1998). Breathing life into a manual: Flexibility and creativity with manual-based treatments. Cognitive and Behavioral Practice, 5, 177-198.

Kendall, P. C., Flannery-Schroeder, E. C., Panichelli-Mindel, S. P., Southam-Gerow, M. A., Henin, A., \& Warman, M. J. (1997). Treating anxiety disorders in youth: A second randomized clinical trial. Journal of Consulting and Clinical Psychology, 65, 366-380.

Kendall, P. C., Hollon, S., Beck, A., Hammen, C., \& Ingram, R. (1987). Recommendations regarding the Beck Depression Inventory. Cognitive Therapy and Research, 11, 289-299.

Kendall, P. C., Kane, M., Howard, B., \& Siqueland, L. (1990). Cognitive-behavioral treatment of anxious children: Treatment manual. Available from the author, Department of Psychology, Temple University, Philadelphia, PA 19122.

Kendall, P. C., \& Southam-Gerow, M. A. (1995). Issues in the transportability of treatment: The case of anxiety disorders in youth. Journal of Consulting and Clinical Psychology, 63, 702-708.

Kendall, P. C., \& Southam-Gerow, M. A. (1996). Long-term followup of a cognitive-behavioral therapy for anxiety-disordered youth. Journal of Consulting and Clinical Psychology, 64, 724730.

Kendall, P. C., \& Sugarman, A. (1997). Attrition in the treatment of childhood anxiety disorders. Journal of Consulting and Clinical Psychology, 65, 883-888.

Kovacs, M. (1992). Children's Depression Inventory Manual. North Tonawanda, NY: Multi-Health Systems.

Lewinsohn, P. M., Clarke, G. N., Rohde, P., \& Hops, H. (1996). A course in coping: A cognitive-behavioral approach to the treatment of adolescent depression. In E. D. Hibbs \& P. S. Jensen (Eds.), Psychosocial treatments for child and adolescent disorders: Empirically based strategies for clinical practice (pp. 109-136). Washington, DC: American Psychological Association.

Loeber, R. (1988). Natural histories of conduct problems, delinquency, and associated substance use: Evidence for developmental progressions. In B. B. Lahey \& A. E. Kazdin (Eds.), Advances in clinical child psychology (Vol. 11, pp. 73-124). New York: Plenum.

Moffitt, T. E. (1993). Adolescence-limited and life-course-persistent antisocial behavior. Psychological Review, 100, 674-701.
Motta, R. W., \& Lynch, C. (1990). Therapeutic techniques vs. therapeutic relationships in child behavior therapy. Psychological Reports, 67, 315-322.

Motta, R. W., \& Tobin, M. I. (1992). The relative importance of specific and nonspecific factors in child behavior therapy. Psychotherapy in Private Practice, 11, 51-61.

Nye, C. L., Zucker, R. A., \& Fitzgerald, H. E. (1995). Early intervention in the path to alcohol problems through conduct problems: Treatment involvement and child behavior change. Journal of Consulting and Clinical Psychology, 63, 831-840.

Ollendick, T. H., \& King, N. J. (1998). Empirically supported treatments for children with phobic and anxiety disorders: Current status. Journal of Clinical Child Psychology, 27, 156-167.

Orlinsky, D. E., \& Howard, K. I. (1986). The psychological interior of psychotherapy: Explorations with the Therapy Session Report Questionnaires. In L. S. Greenberg \& W. M. Pinsof (Eds.), The psychotherapeutic process: A research handbook (pp. 477501). New York: Guilford.

Patterson, G. R., Capaldi, D., \& Bank, L. (1991). An early starter model for predicting delinquency. In D. J. Pepler, \& K. H. Rubin (Eds.), The development and treatment of childhood aggression (pp. 131-168). Hillsdale, NJ: Lawrence Erlbaum Associates, Inc.

Patterson, G. R., \& Chamberlain, P. (1994). A functional analysis of resistance during parent training therapy. Clinical Psychology: Science \& Practice, 1, 53-70.

Phares, V. (1996). Fathers and developmental psychopathology. New York: Wiley.

Phares, V., \& Compas, B. E. (1992). The role of fathers in child and adolescent psychopathology: Make room for daddy. Psychological Bulletin, 111, 387-412.

Rapee, R. M., Barrett, P. M., Dadds, M. R., \& Evans, L. (1994). Reliability of the $D S M-I I I-R$ childhood anxiety disorders using structured interview: Interrater and parent-child agreement. Journal of the American Academy of Child \& Adolescent Psychiatry, 33, 984-992.

Reynolds, C. R., \& Richmond, B. O. (1985). Revised children's manifest anxiety scale (RCMAS): Manual. Los Angeles: Western Psychological Services.

Ronan, K. R., Kendall, P. C., \& Rowe, M. (1994). Negative affectivity in children: Development and validation of a selfstatement questionnaire. Cognitive Therapy and Research, 18, 509-528.

Shirk, S. R., Saiz, C., \& Sarlin, N. (1993, June). The therapeutic alliance in child and adolescent treatment: Initial studies with inpatients. Paper presented at meetings of the Society for Psychotherapy Research, Pittsburgh, PA.

Silverman, W. K. (1991). Anxiety disorders interview schedule for children. Albany, NY: Graywind.

Silverman, W. K., \& Albano, A. M. (1996). Anxiety disorders interview schedule for DSM-IV: Child version. San Antonio, TX: Psychological Corporation.

Silverman, W. K., \& Eisen, A. R. (1992). Age difference in the reliability of parent and child reports of child anxious symptomatology using a structured interview. Journal of the American Academy of Child and Adolescent Psychiatry, 31, $117-124$.

Silverman, W. K., Kurtines, W. M., Ginsburg, G. S., Weems, C. F., White-Lumpkin, P., \& Hicks-Carmichael, D. (1999). Treating anxiety disorders in children with group cognitive-behavior therapy: A randomized clinical trial. Journal of Consulting and Clinical Psychology, 67, 995-1003.

Silverman, W. K., \& Rabian, B. (1995). Test-retest reliability of the $D S M-I I I-R$ childhood anxiety disorders symptoms using the Anxiety Disorders Interview Schedule for Children. Journal of Anxiety Disorders, 9, 139-150.

Silverman, W. K., Saavedra, L. M., \& Pina, A. A. (in press). Test-retest reliability of anxiety symptoms and diagnostic using the 
Anxiety Disorders Interview Schedule for DSM-IV: Child and parent version (ADIS for DSM-IV: C/P). Journal of the American Academy of Child and Adolescent Psychiatry.

Southam-Gerow, M. A., Flannery-Schroeder, E. C., Kendall, P. C. (2001). Psychometric evaluation of the parent-report STAIC. Manuscript submitted for publication.

Southam-Gerow, M. A., \& Kendall, P. C. (2000). Cognitive-behavior therapy with youth: Advances, challenges, and future directions. Clinical Psychology and Psychotherapy, 7, 343366.

Southam-Gerow, M. A., \& Kendall, P. C. (in press). Emotion regulation and understanding: Implications for child psychopathology and therapy. Clinical Psychology Review.

Spielberger, C. (1973). Manual for the State-Trait Anxiety Inventory for Children. Palo Alto, CA: Consulting Psychologists Press.

Spielberger, C., Gorusch, R., \& Lushene, R. (1970). STAI manual. Palo Alto, CA: Consulting Psychologists Press.

Stanger, C., \& Lewis, M. (1993). Agreement among parents, teachers, and children on internalizing and externalizing behavior problems. Journal of Clinical Child Psychology, 22, 107-115.

Stoolmiller, M., Duncan, T., Bank, L., \& Patterson, G. R. (1993). Some problems and solutions in the study of change: Significant patterns in client resistance. Journal of Consulting and Clinical Psychology, 61, 920-928.

Strauss, C. (1987). Modification of trait portion of State-Trait Anxiety Inventory for Children-Parent Form. (Available from Cyd C. Strauss, University of Florida, Department of Psychology, P.O. Box 100165, Gainesville, FL 32610-0165).

Tabachnick, B. G., \& Fidell, L. S. (1996). Using multivariate statistics. New York: HarperCollins.
Treadwell, K. R. H., Flannery-Schroeder, E. C., \& Kendall, P. C. (1995). Ethnicity and gender in relation to adaptive functioning, diagnostic status, and treatment outcome in children from an anxiety clinic. Journal of Anxiety Disorders, 9, 373-384.

Webster-Stratton, C. (1985). Predictors of treatment outcome in parent training for conduct disordered children. Behavior Therapy, $16,223-243$.

Weisz, J. R. (2000). Lab-clinic differences and what we can do about them: I. The Clinic-Based Treatment Development Model. Clinical Child Psychology Newsletter, 15(1), 1-3, 10.

Weisz, J. R., \& Weersing, V. R. (1998). Developmental outcome research. In W. K. Silverman \& T. H. Ollendick (Eds.), Developmental issues in the clinical treatment of children and adolescents (pp. 457-469). Needham Heights, MA: Allyn \& Bacon.

Weisz, J. R., Weiss, B., Han, S. S., \& Granger, D. A. (1995). Effects of psychotherapy with children and adolescents revisited: A meta-analysis of treatment outcome studies. Psychological Bulletin, 117, 450-468.

Zahn-Waxler, C., Kochanska, G., Krupnick, J., \& McKnew, D. (1990). Patterns of guilt in children of depressed and well mothers. Developmental Psychology, 26, 51-59.

Manuscript received June 6, 2000

Final revision received February 16, 2001 